\title{
Influence of Amalgam, Alloy, and Mercury on the In Vitro Growth of Streptococcus mutans: II. Comparison of Amalgams and Alloys
}

\author{
LOYS J. NUNEZ,* GOTTFRIED SCHMALZ,† JOHN HEMBREE, and \\ LESTER D. HULETT, JR.‡ \\ Department of Biomaterials, College of Dentistry, University of Tennessee \\ Center for the Health Sciences, Memphis, Tennessee 38163, USA
}

The influence of three alloys and their amalgams on the in vitro growth of Streptococcus mutans has been estimated. Spherical, fine cut, and dispersion alloys were studied. Dispersion alloy amalgams seem to inhibit bacterial growth more than amalgams prepared from the other alloys in a similar fashion.

A biologic test system has already been described 1 to estimate the effect of amalgams and alloys on the growth of Streptococcus mutans in vitro. This test system has been used to study three alloys and amalgams prepared from these alloys. The alloys include a spherical alloy, a fine cut alloy, and a dispersion alloy, representing materials of different chemical compositions and physical properties. The objective of this work was to determine what effect, if any, amalgams prepared from these alloys have on the in vitro growth of $S$ mutans. All of the alloys studied are in use in dental practice today.

Reports in the literature have been conflicting as to whether silver amalgams are antibacterial. 2-4 Perhaps some of these conflicting observations may be better understood in terms of the results presented here.

\section{Materials and Methods}

The test system has previously been de-

This investigation was supported, in part, by Grant No. 2434704606-R74 from the L. G. Noel Memorial Fund.

Received for publication August 28, 1975.

Accepted for publication March 19, 1976.

- Dr. Nunez is also with Materials Science Toxicology Laboratories, College of Pharmacy, University of Tennessee Center for the Health Sciences, Memphis.

† Permanent address: ZMK Klinik, 74 Tubingen, Osianderstrasse 2-8, W Ger.

$\ddagger$ Dr. Hulett is with the Oak Ridge National Laboratory, Oak Ridge, Tenn. scribed in detail. ${ }^{1}$ Triple-distilled mercury was used in all amalgamations. ${ }^{a}$ Spherical alloy, ${ }^{b}$ fine cut alloy, ${ }^{c}$ and a dispersion alloy were used in pellet form.

The alloy was used unaltered as it came from the producer. Appropriate amounts were obtained by cutting the pellets. The sterilization procedure was the same as for amalgams.

The alloy powders shown in the photomicrographs were obtained by placing two alloy pellets in the trituration apparatus for 18 seconds.

Mercury was added to the alloy by means of dispensers.e,f,g The dispensers were adjusted to deliver $50 \%$ mercury. For all amalgams, trituration was performed mechanically for 18 seconds with two pellets and the appropriate amount of mercury in the capsule.

After trituration all samples were prepared as described previously. ${ }^{1}$

A batch contained metal samples and con-

a Rx Mercury, ACS-VSP-CP-NF, Codesco Inc., Philadelphia, Pa.

b Spheraloy, Kerr Manufacturing Co., Romulus, Mich. Del.

c Caulk Alloy Fine Cut, L. D. Caulk Co., Milford,

d Dispersalloy, Johnson \& Johnson, Dental Products Co., East Windsor, NJ.

- Caulk Mercury Dispenser, L. D. Caulk Co., Milford, Del.

1 Dispersalloy Tablet and Mercury Dispenser, Johnson \& Johnson Dental Products Co., East Windsor, NJ.

g Kerr Spheraloy Proportioner, Kerr Manufacturing Co., Detroit, Mich.

h Wig-L-Bug Amalgamator, Crescent Dental Mfg. Co., Chicago, Ill. 


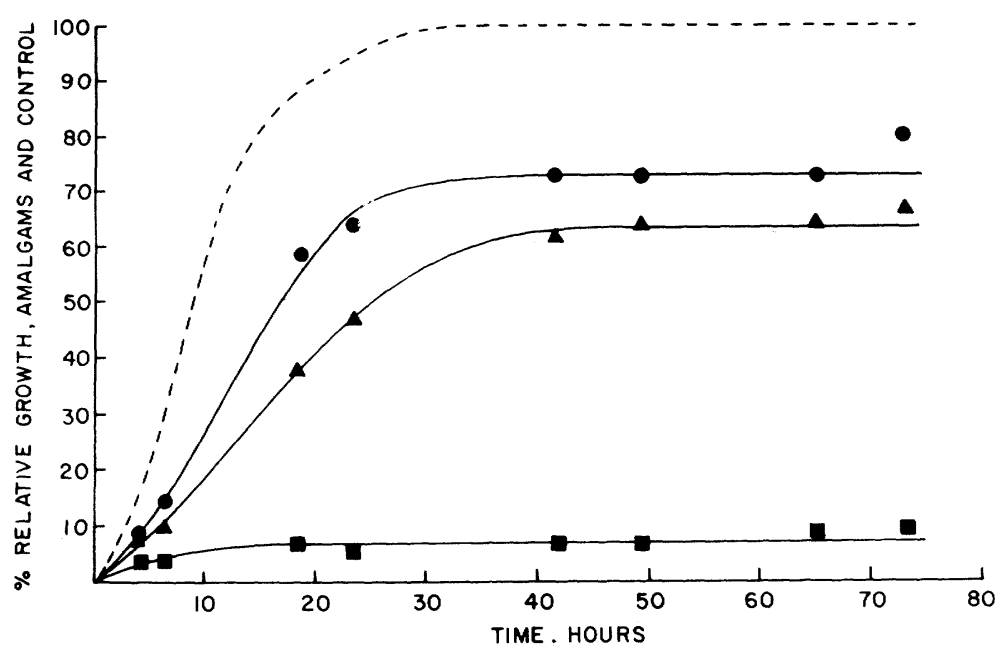

Fig 1.-Influence of different amalgams on growth. Sample wt/test tube, 1.56 gm. - - , spherical amalgam; $-\mathbf{\Delta}-$, fine cut amalgam; $-\mathbf{-}-$, dispersion amalgam; and ..... inoculated control (no metal).

trols prepared at the same time and all inoculated from the same generation of the bacterial strain. Batches have frequently been prepared with inoculation from the same bacterial strain for a period of about a year.

Four types of samples were prepared for each batch: (l) medium, no bacteria, no metals; (2) medium plus bacteria, no metals;
(3) medium plus metal, no bacteria; and (4) medium plus metal, plus bacteria.

Types 1, 2, and 3 served as controls. For each alloy or amalgam studied, five replicate samples were prepared corresponding to type 4. For every batch, five replicate samples corresponding to type 2 were run as controls. The spectrophotometric readings for the five samples were averaged and the growth of

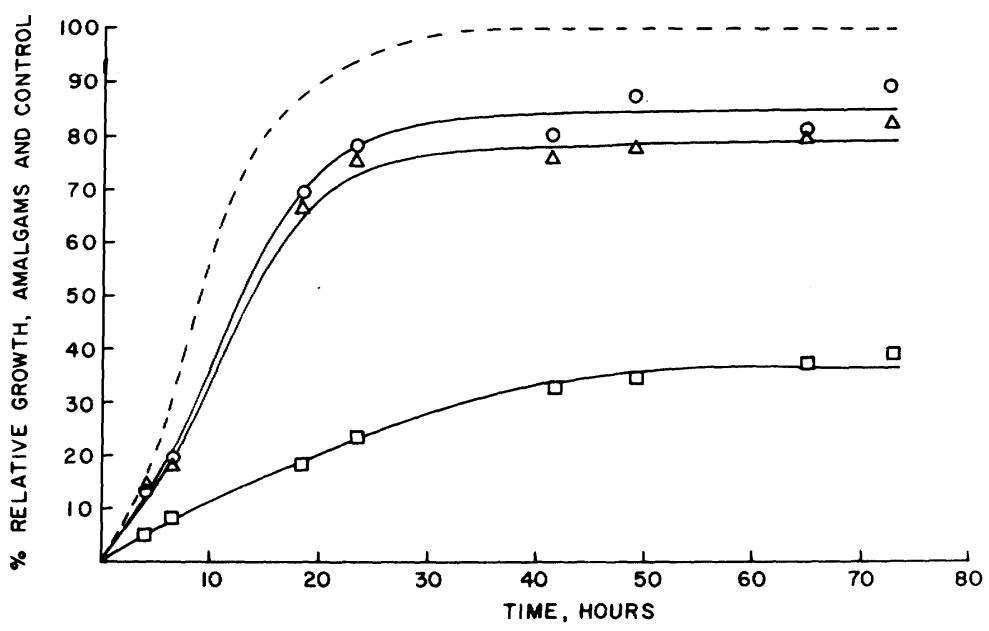

Fig 2.-Influence of different amalgams on growth. Sample wt/test tube $0.78 \mathrm{gm}$. - $\bigcirc-$, spherical amalgam; $-\triangle-$, fine cut amalgam; $-\square-$, dispersion amalgam; -...-, inoculated control ( no metal). 
bacteria in the presence of alloys or amalgams expressed as the percent final growth of the controls as follows:

$$
\%_{\mathrm{o}} R A_{\mathrm{t}}=\frac{\Delta A_{\mathrm{t}} \times 100}{\Delta C_{\mathrm{f}}},
$$

where $\% R A_{\mathrm{t}}$ is growth of amalgam or alloy relative to controls at time, $\mathrm{t} ; \Delta A_{\mathrm{t}}$, increased bacterial growth at time, $t$, above initial inoculation for alloys or amalgams corrected for changes in the medium simultaneously taking place; and $\Delta C_{\mathrm{f}}$, final bacterial growth of controls (no metal).

Relative growth curves are seen in Figures 1 and 2. It can be seen from these curves that a plateau is reached in the vicinity of 45 to 75 hours. Many other plots not shown here also demonstrate that a plateau usually occurs in the vicinity of 45 to 75 hours. The relative growth of the bacteria at 60 hours, designated $\% R A_{60}$, is taken as a measure of the effect on growth resulting from contact with the alloy or amalgam.

Normal growth of controls is usually completed after approximately 24 hours of incubation. A few instances have been observed in which a 15- or 20-hour delay in initiation of control growth has taken place with subsequent slower than normal growth. These batches were discarded.

The initial percent transmittance reading of uninoculated controls (with metal samples) is usually in the vicinity of 56 to $60 \%$. After 75 hours of incubation, this value will normally change by no more than a few percent. Should great changes occur in this control on incubation, the method would not be applicable. No such instances have been observed in this study.

Statistical tests used in comparing means were of the form corresponding to the un. paired $t$ test for the difference between means:

or

$$
t=\frac{\text { Observed }- \text { Expected }}{\text { Appropriate Standard Deviation }}
$$

$$
t=\frac{(\bar{X}-\bar{Y})-a}{S_{\bar{x}-\bar{y}}},
$$

where $\bar{X}$ and $\bar{Y}$ represent observed means; $S_{\bar{x}-\bar{y}}$, the estimated standard deviation based on a pooled variance; and $a$, the corresponding difference in population means. The null hypothesis may be set up such that

$$
\text { Ho: } \mu_{x}-\mu_{y}=a,
$$

where $\mu_{x}$ and $\mu_{y}$ represent population means.

When $X, Y$, and $S_{x-y}$ are known, $t$ may be obtained from the table for the appropriate degrees of freedom and $\alpha$-level and the aforementioned equation may be solved for $a$.

Mean values of $a$ allowed an estimate to be made of the reproducibility between batches.

Analysis of variance treatment was used to estimate significant differences between means within a batch. A computer program obtained from the UCLA Health Sciences Computing Facility and later modified at Memphis State University was used for analysis of variance calculations. 1 Multiple-range tests and the Student-Newman-Keuls procedure compared mean pairs for significant differences.

\section{Results}

The reproducibility of results was estimated both within batches and between batches, many observations suggesting that there was less variation within batches than between batches.

Duplicate growth curves obtained for the same alloy, each curve coming from a different batch, allowed an estimate to be made of between-batch reproducibility. Eight such pairs of curves included the three types of alloys in amounts in the test tubes considered to be in the normal range. Mean values were calculated in the plateau region of the curves bracketing the $\% R A_{60}$ value. It was found that the batch-to-batch difference between two $\% R A_{60}$ means, corresponding to duplicate runs on the same alloy, is no greater than $14 \%$ at the $\alpha=0.05$ level. This implies that two alloy samples must differ

\footnotetext{
1 Xerox Sigma 9 Computer, Memphis State University Computer Services, Memphis, Tenn.
}

TABLE

Approximate Elemental Analysis of Alloys

\begin{tabular}{lccc}
\hline \hline & $\begin{array}{c}\text { Spherical } \\
\text { Alloy (\%) }\end{array}$ & $\begin{array}{c}\text { Fine Cut } \\
\text { Alloy (\%) }\end{array}$ & $\begin{array}{c}\text { Dispersion } \\
\text { Alloy (\%) }\end{array}$ \\
\hline $\mathrm{Ag}$ & 52 & 60 & 35 \\
$\mathrm{Sn}$ & 26 & 29 & 17 \\
$\mathrm{Cu}$ & 21 & 5 & 42 \\
$\mathrm{Zn}$ & 0.7 & 5 & 4 \\
$\mathrm{~Pb}$ & 0.1 & 1 & 2 \\
Other & 0.2 & $\ldots$ & $\ldots$ \\
\hline
\end{tabular}




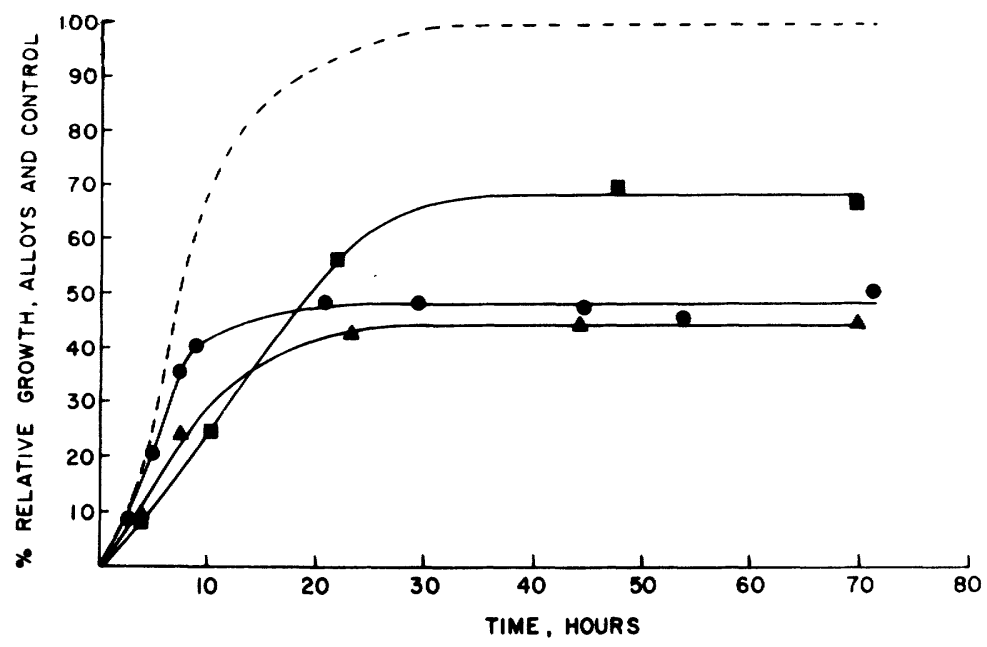

Fig 3.-Influence of different alloys on growth. Sample wt/test tube 0.78 gm. - - - spherical alloy; $-\mathbf{\Delta}-$, fine cut alloy; $-\mathbf{-}-$, dispersion alloy; and -.---, inoculated control (no metal).

from one another in growth inhibition by more than $10 \%$ before the method can distinguish a difference between the alloys at the $95 \%$ confidence level (on the basis of duplicate runs, each from a different batch) The value of $10 \%$ corresponds to the average difference between two population means of $\% R A_{60}$ values each coming from a different batch.

Relative growth curves for amalgams prepared from a fine cut alloy, ${ }^{c}$ a spherical alloy, ${ }^{b}$ and a dispersion phase alloyd are seen in Figure 1, where three curves represent equal weights of amalgam (1.56 gm) in the test tube. The control growth (no metal) curve is also shown. Each point plotted in Figure 1 represents an average of five measurements. It can be seen that the dispersion phase amalgam inhibits growth of $S$ mutans more than the fine cut and spherical amalgams as indicated by the lower percent relative growth values in the vicinity of 60 hours. Analysis of variance and multiplerange tests indicate that all three amalgams

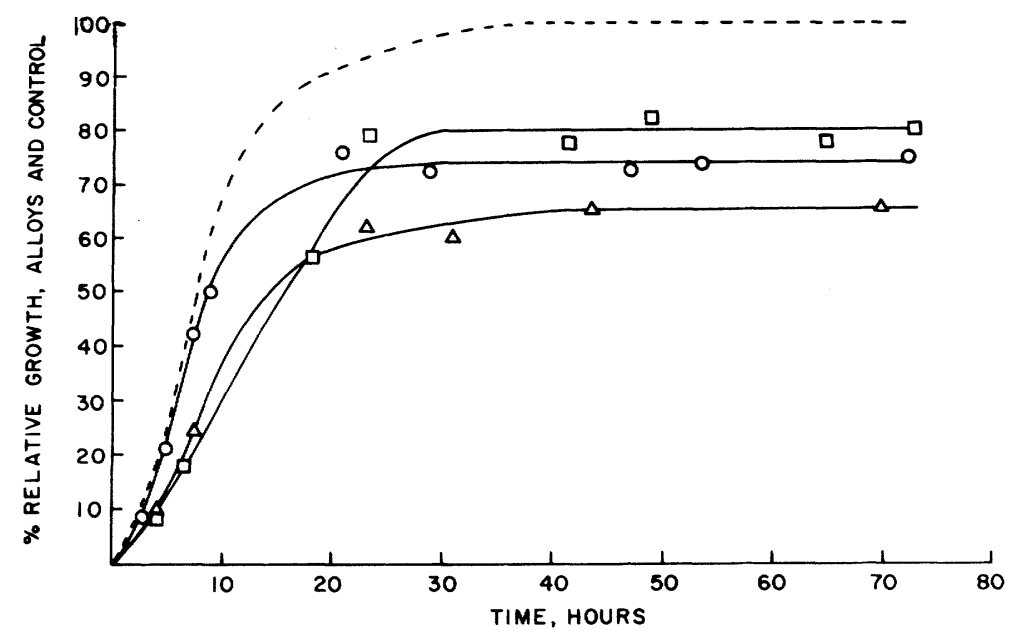

Fig 4.-Influence of different alloys on growth. Sample wt/test tube 0.39 gm. $-\bigcirc-$, spherical alloy; $-\Delta-$, fine cut alloy; $-\square-$, dispersion alloy; and -...-, inoculated control (no metal). 


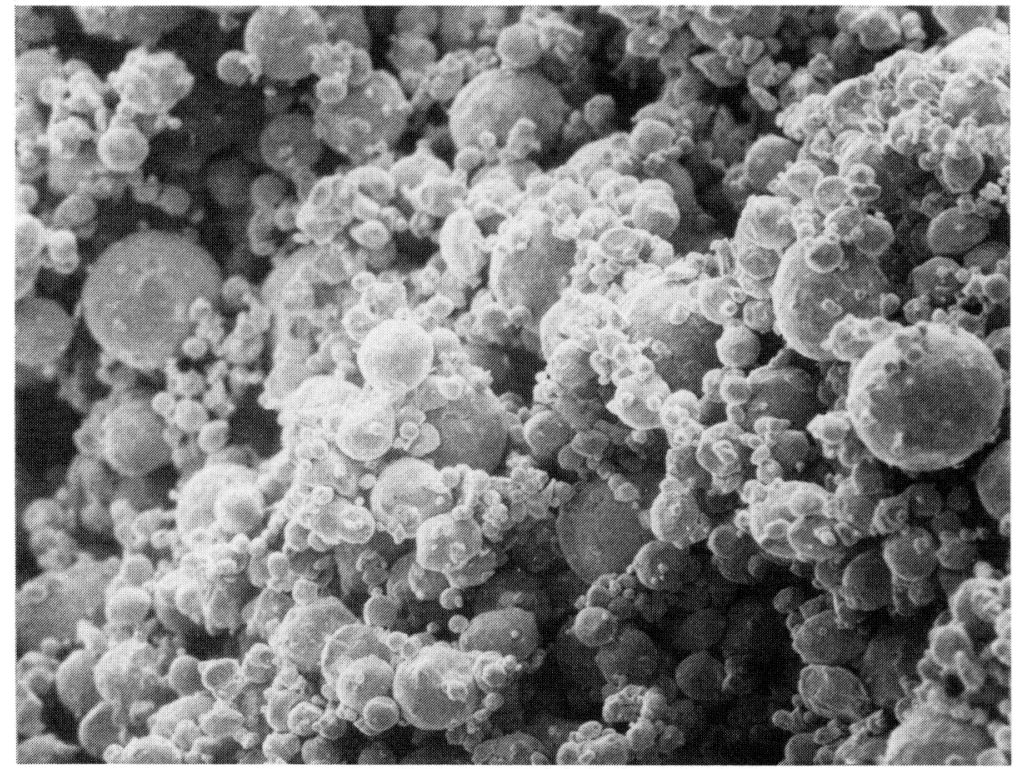

FIG 5.-Spherical alloy $(\mathrm{mag} \times 700)$.

are significantly different in growth inhibition at the $\alpha=0.05$ level.

Similar curves are seen in Figure 2 where three curves represent equal but smaller weights of amalgam $(0.78 \mathrm{gm})$ in the test tube. The $\%_{0} R A_{60}=86.2$ for the amalgam prepared from the spherical alloy and is not significantly different from the $\% R A_{60}=$ 79.8 for the amalgam prepared from the fine cut alloy at the $\alpha=0.05$ level. Thus, it may be concluded that amalgams prepared from the spherical alloy and the fine cut alloy show about the same growth inhibition. However, amalgams prepared from the dispersion alloy showed greater inhibition to growth of $S$ mutans. These conclusions are based on comparisons made within the same batch.

Analysis of variance of the data seen in Figures 1 and 2 confirmed our assumption that the relative growth curves for $S$ mutans had reached a plateau in the vicinity of 60 hours. For any given curve, the $\% R A_{\mathrm{t}}$ mean values were not significantly different from one another at the $\alpha=0.05$ level in the time range of 45 to 75 hours. Thus, it would appear that the $\% R A_{60}$ value is an adequate measure of growth effect. Comparison of the means for variables other than time, however, show that the null hypothesis of equality of population means must be rejected at the $\alpha=0.05$ level.
The curves of Figure 3 show relative growth curves of $S$ mutans for the three alloys. Three curves represent equal weights of alloy $(0.78 \mathrm{gm})$ and the control growth curve is also shown. There is little difference in growth inhibition between the spherical alloy and the fine cut alloy, but they both inhibit growth of $S$ mutans significantly more than the dispersion phase alloy.

Similar curves are seen in Figure 4 for equal weights of alloy of $0.39 \mathrm{gm}$. Analysis of variance indicates that all three plateau regions of the curves are significantly different from one another at the $\alpha=0.05$ level.

The data plotted in Figures 3 and 4 show the same trend for the two different weights of alloys in the test tubes: the dispersion alloy inhibits growth less than the spherical alloy which in turn inhibits growth less than the fine cut alloy.

The scanning electron micrographs ${ }^{j}$ of Figures 5, 6, and 7 demonstrate the different physical forms of the alloys. The magnification is $\times 700$.

The elemental composition of the three alloys was estimated by means of X-ray fluorescence. These data are given in the table. The error in determining the metals of high

1 Obtained by Dr. Bill Becker, department of chemical and metallurgical engineering, University of Tennessee, Knoxville, Tenn. 


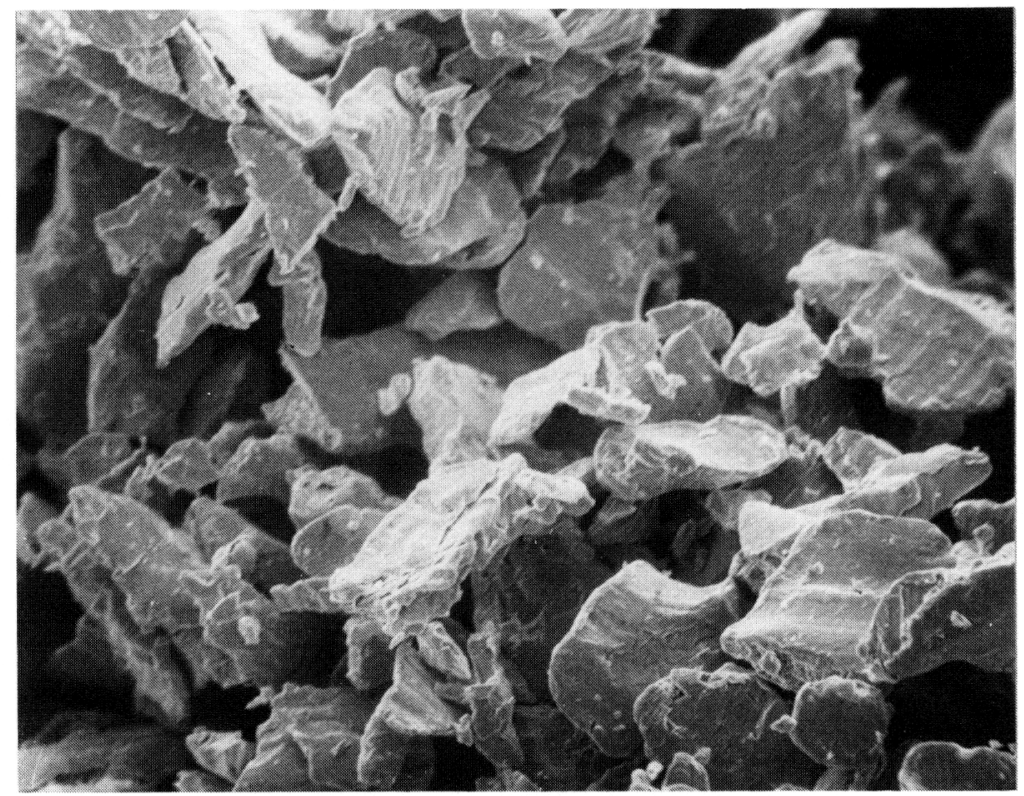

FIG 6.-Fine cut alloy (mag $\times 700)$.

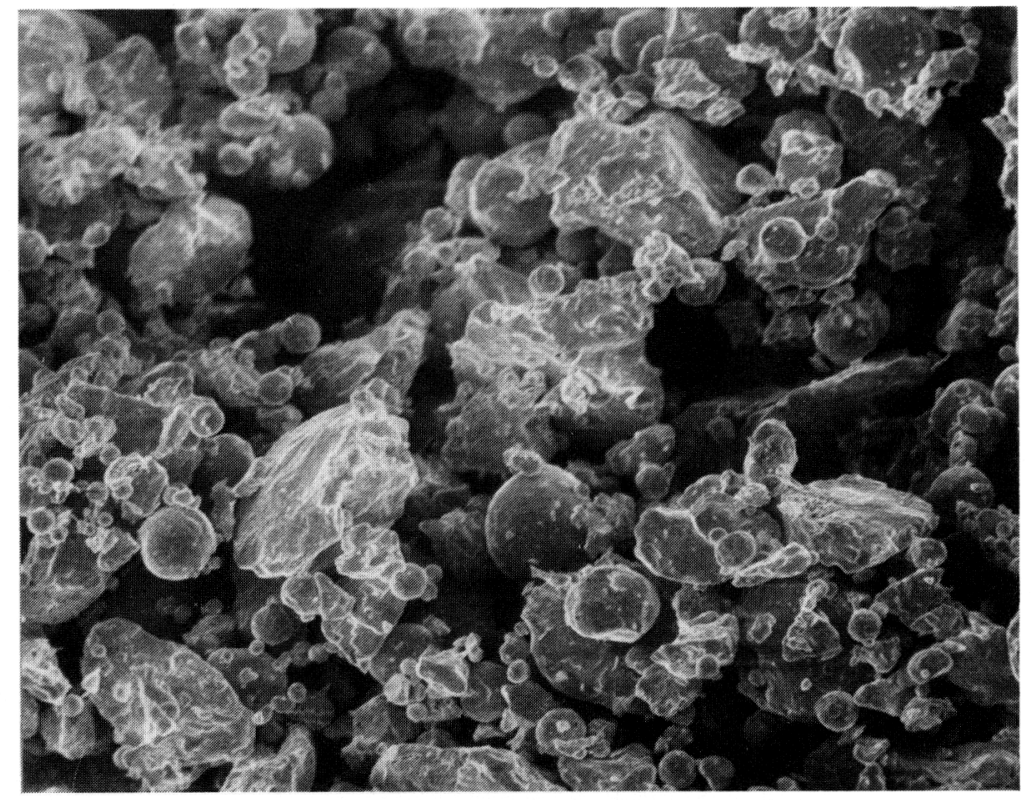

FIG 7.-Dispersion alloy $(\operatorname{mag} \times 700)$. 
composition ( $\mathrm{Ag}, \mathrm{Sn}, \mathrm{Cu}$ ) should be no greater than $15 \%$. The error in the elements of low composition ( $\mathrm{Zn}$ and $\mathrm{Pb}$ ) could be as high as $50 \%$.

\section{Discussion and Conclusions}

It is evident from Figures 3 and 4 that the dispersion phase alloy inhibits $S$ mutans growth less than the other two alloys. The chemical composition of the alloys as given in the table seems to explain the order of inhibition reasonably well. The ratio of $\mathrm{Ag}$ to $\mathrm{Sn}$ is approximately $2: 1$ in all of the alloys. The total amount of $\mathrm{Ag}$ or $\mathrm{Sn}$ or both in the alloys may be ranked as: fine cut $>$ spherical $>$ dispersion alloy. The ranking in terms of inhibition of bacterial growth is in precisely the same order.

Amalgams prepared from the dispersion alloy inhibit growth of $S$ mutans more than amalgams prepared from the other alloys, however, as shown in Figures 1 and 2. What, then can account for this change of rank in going from alloy to amalgam? It would seem that differences in elemental analysis as given in the table cannot account for this effect per se. However, phase composition of amalgams prepared from alloys of these elemental compositions may vary considerably. The phase composition and changes in phase composition with time of dispersion alloy amalgams have been discussed by Sarkar. ${ }^{5}$ We can only speculate that the initial phase composition of dispersion phase amalgams may account for greater growth inhibition. Perhaps a particular phase may be consider. ably more toxic than another. Future work may confirm or deny some of these specula. tions.

A recent publication reported cytotoxicity data on spherical and fine cut amalgams in contact with $\mathrm{L}$ strain fibroblasts derived from subcutaneous tissue of $\mathrm{C} 3 \mathrm{H}$ strain mice. ${ }^{6}$ Persistent differences were found in cytotoxicity between spherical and fine cut amalgams even with the same alloy-mercury ratio, which in this instance was 5:8. All of our amalgams had alloy-mercury ratios of 5:5 as recommended by the manufacturer so that direct comparison with Kawahara et al's ${ }^{6}$ work is not possible. Also Kawahara et $a^{6}$ did not report data on dispersion phase amalgams.

There remains the interesting possibility that some materials may possess low cytotoxicity as well as high bacterial growth inhibition. Such amalgams, if they exist with desirable physical properties, could possibly be advantageous in a clinical situation.

\section{References}

1. Nunez, L.J.; Schmalz, G.; and Hembree, J.; Influence of Amalgam, Alloy and Mercury on the In Vitro Growth of Streptococcus mutans: I. Biological Test System, J Dent Res 55: 257 261, 1976.

2. Noonan, R.G.: Silver Amalgam is Not AntiBacterial, J Dent Child 32: 147-153, 1965.

3. Shay, D.E.; Allen, T.J.; and Mantz, R.F.: The Antibacterial Effects of Some Dental Restorative Materials, J Dent Res 35: 25-33, 1956.

4. McCue, R.W.; McDougal, F.G.; and Shary, D.E.: The Antibacterial Properties of Some Dental Restorative Materials, Oral Surg 4: 1180-1184, 1951.

5. SARKAR, N.K.: The Electrochemical Behavior of Dental Amalgams and Their Component Phases, PhD dissertation in biological materials, Northwestern University, Evanston, Ill, 1973.

6. Kawahara, H.; Nakamura, M.; Yamagami, A.; and Nakanishi, T.: Cellular Responses to Dental Amalgam In Vitro, $J$ Dent Res 54: 394 $401,1975$. 